\title{
A relaxed growth modeling framework for controlling growth-induced residual stresses
}

\author{
M. Genet ${ }^{\mathrm{a}, \mathrm{b}, *}$ \\ ${ }^{a}$ Laboratoire de Mécanique des Solides, École Polytechnique / CNRS / Université \\ Paris-Saclay, Palaiseau, France \\ ${ }^{b}$ M3DISIM team, INRIA / Université Paris-Saclay, Palaiseau, France
}

\begin{abstract}
(Abstract word count: 245.)

Background. Constitutive models of the mechanical response of soft tissues have been established and are widely accepted, but models of soft tissues remodeling are more controversial. Specifically for growth, one important question arises pertaining to residual stresses: existing growth models inevitably introduce residual stresses, but it is not entirely clear if this is physiological or merely an artifact of the modeling framework. As a consequence, in simulating growth, some authors have chosen to keep growth-induced residual stresses, and others have chosen to remove them.

Methods. In this paper, we introduce a novel "relaxed growth" framework allowing for a fine control of the amount of residual stresses generated during tissue growth. It is a direct extension of the classical framework of the multiplicative decomposition of the transformation gradient, to which an additional sub-transformation is introduced in order to let the original unloaded configuration evolve, hence relieving some residual stresses. We provide multiple illustrations of the framework mechanical response, on time-driven constrained growth as well as the strain-driven growth problem of the artery under internal pressure, including the opening angle experiment.

Findings. The novel relaxed growth modeling framework introduced in this paper allows for a better control of growth-induced residual stresses compared to standard growth models based on the multiplicative decomposition of the transformation gradient.
\end{abstract}

\footnotetext{
*martin.genet@polytechnique.edu
} 
Interpretation. Growth-induced residual stresses should be better handled in soft tissues biomechanical models, especially in patient-specific models of diseased organs that are aimed at augmented diagnosis and treatment optimization.

Keywords:

Finite growth, Residual stresses, Relaxation, Finite element method

(Total word count: ca. 3000.)

\section{Introduction}

Biomechanical models are widely considered as good candidates to improve patient diagnosis and treatment of various diseases, for instance in vascular [Taylor and Figueroa, 2009], cardiac [Smith et al., 2011; Krishnamurthy et al., 2013], or respiratory [Roth et al., 2017] mechanics. To do so, these models must first be formulated with patient-agnostic constitutive laws and parameters that are developed alongside with experiments. And once these generic models are set up, clinical data must be assimilated into to personalize them and hence produce diagnosis [Xi et al., 2011; Genet et al., 2015a] and/or perform in silico treatment optimization [Sermesant et al., 2012].

Today, most personalized modeling pipelines focus on the current state of the tissue/organ, and few work focused on predicting the long term evolution of the system of interest, i.e., prognosis [Clatz et al., 2005; Rausch et al., 2017]. Similarly, constitutive models of the mechanical response of soft tissues have been established and are widely accepted in the community, but models of soft tissues remodeling are more controversial [Witzenburg and Holmes, 2017]. This is explained by the increased complexity and associated decreased understanding of the physical mechanisms at play during remodeling. Specifically for growth, a remodeling mechanism associated with the addition and/or removal of matter with unchanged properties [Taber, 1995; Kuhl, 2014], there are competing approaches for the very description of growth (multiplicative decomposition of the transformation gradient [Rodriguez et al., 1994; Kuhl, 2014] vs. constrained mixture theory [Humphrey and Rajagopal, 2002; Valentín and Holzapfel, 2012; Cyron et al., 2016]), the growth driving force (chemistry vs. mechanics [Maillet et al., 2013; Kuhl, 2014], strain vs. stress [Rodriguez et al., 1994; Göktepe et al., 2010; Kerckhoffs et al., 2012], static vs. oscillatory loading [Maillet et al., 2013; Lee et al., 
2015], etc.). Nevertheless, the next generation of personalized modelingbased clinical tools might include remodeling mechanisms such as growth for prognosis and treatment optimization.

One important question associated with the modeling of growth is about the induced residual stresses, or equivalently, prestrain. On the one hand they are present in living tissues [Fung, 1993], on the other hand growth naturally induces residual stresses [Rodriguez et al., 1994; Skalak et al., 1996]; however it is still largely unknown how much of the residual stresses induced by the physiological or pathological growth remain, and how much are relaxed away by some relaxation mechanism [Fung, 1993; Taber, 1995]. Actually, it was found experimentally, in studies involving hypertension-induced pathological growth of cardiovascular tissues, that opening angle, and hence residual stresses, were only very little correlated with growth [Liu and Fung, 1989; Omens et al., 1996]. As a consequence, in growth models of the literature, some authors have chosen to keep growth-induced residual stresses [Rodriguez et al., 1994; Lee et al., 2014; Genet et al., 2015b], and others have chosen to remove them [Kroon et al., 2009; Lee et al., 2015, 2016]. Other approaches have been proposed to better deal with growth-induced residual stresses, notably a recent growth modeling framework that include some "fluid-like" growth [Böl and Bolea Albero, 2014; Bolea Albero et al., 2014].

In this paper, we introduce a novel "relaxed growth" model, in the general framework of the multiplicative decomposition of the transformation gradient, which allows one to control the amount of growth-induced residual stresses. The general idea is to add another sub-transformation in the decomposition, which relaxes the tissue by transforming its unloaded configuration. Illustrations are provided on time-driven constrained growth as well as the strain-driven growth problem of the artery under internal pressure.

\section{Methods}

\subsection{Review of classical growth modeling}

\subsubsection{Kinematics}

In this part, we review the standard formulation of finite growth modeling based on the multiplicative decomposition of the transformation gradient, originally proposed by [Rodriguez et al., 1994], and illustrated on Figure 1. Thus, let us consider a physical body $\mathcal{B}$ that initially occupies a domain $\Omega_{0}$ and that, after deformation and growth, occupies a domain $\Omega$. The geometrical transformation between material neighborhoods of $\mathcal{B}$ in $\Omega_{0}$ and 


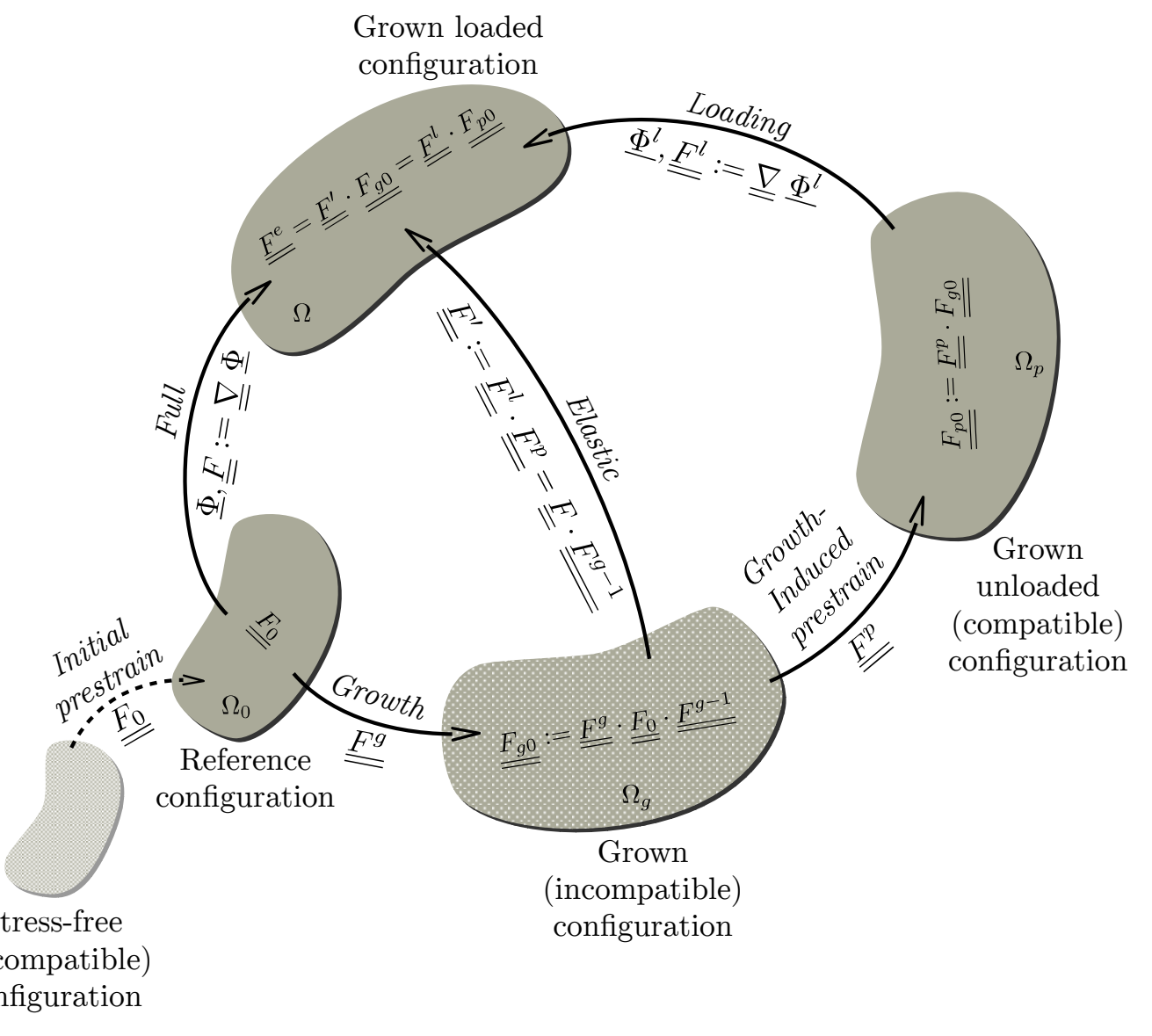

Figure 1: Schematic of the multiplicative decomposition of the transformation gradient into growth, prestrain and loading parts, revealing the elastic part of the transformation [Rodriguez et al., 1994]. Because the growth part represents the transformation of material neighborhoods independently from each others and hence is incompatible (i.e., does not derive from a continuous mapping), the grown configuration is incompatible (i.e., discontinuous everywhere). Consequently, the prestrain and elastic parts of the transformation are also incompatible. Conversely, both the full transformation and the loading part of the transformation are compatible, though if the loading part does represent a continuous mapping between material points, the full transformation only represents a continuous mapping between material neighborhoods, in which mass has potentially been added or removed. This decomposition is generally represented without initial prestrain, i.e., with $\underline{\underline{F_{0}}}=\underline{\underline{1}}$, in which case $\underline{\underline{F_{g 0}}}=\underline{\underline{1}}$ and $\underline{\underline{F_{p 0}}}=\underline{\underline{F^{p}}}$, such that $\underline{\underline{F^{e}}}=\underline{\underline{F^{\prime}}}$. 
$\Omega$ is denoted $\underline{\Phi}$. Following the classical scheme of Figure 1, the transformation gradient $\underline{\underline{F}}:=\underline{\operatorname{Grad}}(\underline{\Phi})$ is multiplicatively decomposed into growth, prestrain, and load-induced parts:

$$
\underline{\underline{F}}=\underline{\underline{F^{l}}} \cdot \underline{\underline{F^{p}}} \cdot \underline{\underline{F^{g}}}
$$

4 where, in general, both $\underline{\underline{F^{g}}}$ (the internal variable describing tissue growth) and $\underline{\underline{F^{p}}}$ (the prestrain) are incompatible second order tensor fields, and $\underline{\underline{F^{l}}}:=\underline{\underline{G r a d}}\left(\underline{\Phi^{l}}\right)$ is the gradient of the (compatible) transformation induced by mechanical loading. The prestrain and load-induced parts can be multiplicatively combined to form the elastic part of the transformation:

$$
\underline{\underline{F^{\prime}}}:=\underline{\underline{F^{l}}} \cdot \underline{\underline{F^{p}}}
$$

such that the full transformation gradient can be multiplicatively decomposed into growth and elastic parts:

$$
\underline{\underline{F}}=\underline{\underline{F^{\prime}}} \cdot \underline{\underline{F^{g}}}
$$

Equivalently, the elastic part of the transformation can be expressed from the full transformation gradient and the growth internal variable:

$$
\underline{\underline{F^{\prime}}}=\underline{\underline{F}} \cdot \underline{\underline{F^{g-1}}}
$$

The full right Cauchy-Green dilatation tensor is denoted $\underline{\underline{C}}:={ }^{t} \underline{\underline{F}} \cdot \underline{\underline{F}}$. As for the transformation gradient, the elastic part of the dilatation tensor can be expressed from the full dilatation tensor and the growth internal variable:

$$
\underline{\underline{C^{\prime}}}:={ }^{t} \underline{\underline{F^{\prime}}} \cdot \underline{\underline{F^{\prime}}}={ }^{t} \underline{\underline{F}}^{g-1} \cdot \underline{\underline{C}} \cdot{\underline{\underline{F^{g-1}}}} .
$$

\subsubsection{Free energy and Stresses}

The elastic response is governed by the strain energy potential, $W^{e}$. Any hyperelastic potential can be used. The main modeling assumption is that in fine the free energy $\rho_{0} \Psi$ is not a function of the total transformation but only its elastic part $\underline{\underline{F^{e}}}$ [Rodriguez et al., 1994; Göktepe et al., 2010]:

$$
\rho_{0} \Psi\left(\underline{\underline{C}}, \underline{\underline{F^{g}}}\right)=W^{e}\left(\underline{\underline{C^{e}}}:={ }^{t} \underline{\underline{F^{e}}} \cdot \underline{\underline{F^{e}}}\right) .
$$

22 Consequently, the second Piola-Kirchhoff stress tensor can be expressed as

$$
\underline{\underline{\Sigma}}=\frac{\partial \rho_{0} \Psi}{\partial \underline{\underline{E}}}=2 \frac{\partial \rho_{0} \Psi}{\partial \underline{\underline{C}}}=2 \frac{\partial W^{e}}{\partial \underline{\underline{C^{e}}}}: \frac{\partial \underline{\underline{\underline{C}}}}{\partial \underline{\underline{C}}} .
$$


2 such that

and

In the canonical case described so far, we have

$$
\underline{\underline{F^{e}}}=\underline{\underline{F^{\prime}}}=\underline{\underline{F}} \cdot{\underline{\underline{F^{g-1}}}}
$$

$$
\underline{\underline{C^{e}}}=\underline{\underline{C^{\prime}}}={ }^{t}{\underline{\underline{F^{g-1}}}} \cdot \underline{\underline{C}} \cdot \underline{\underline{F^{g-1}}},
$$

$$
\underline{\underline{\Sigma}}=\underline{\underline{F^{g-1}}} \cdot 2 \frac{\partial W^{e}}{\partial \underline{\underline{C^{e}}}} \cdot{ }^{t} \underline{\underline{F^{g-1}}} .
$$

4 2.1.3. Growth evolution law

An evolution law must be formulated for the growth tensor to close the system. Several types have been proposed, including simple rates [Kuhl, 2014], strain- [Göktepe et al., 2010; Kerckhoffs et al., 2012; Genet et al., 2016] and stress- [Göktepe et al., 2010] driven laws. See for instance [Kuhl, 2014] for a recent review, and [Witzenburg and Holmes, 2017] for a detailed comparison of existing laws.

In case of tissues subjected to cyclic loading, it is necessary to uncouple the temporal scales of loading and remodeling, by alternating between loading (i.e., fast time scale) and growth (i.e., slow time scale) steps [Kerckhoffs et al., 2012; Lee et al., 2016]. Thus, for a given growth step, the driving force for growth must be computed from the previous loading step. Average [Lee et al., 2015] and maximum [Kerckhoffs et al., 2012] values of stress and strain over a cycle have been proposed, in agreement with experimental analysis [Holmes, 2004].

\subsubsection{Case of initially prestrained material}

For the sake of completeness, let us consider the case where the initial configuration $\Omega_{0}$ has some potentially incompatible initial prestrain, denoted $\underline{\underline{F_{0}}}$, or, equivalently, some autobalanced initial prestress $\underline{\underline{\sigma_{0}}}=\frac{1}{J_{0}} \underline{\underline{F_{0}}} \cdot \underline{\underline{\Sigma_{0}}} \cdot \underline{\underline{F_{0}}}{ }^{-1}$ with $J_{0}:=\operatorname{det}\left(\underline{\underline{F_{0}}}\right)$ and $\underline{\underline{\Sigma_{0}}}=2 \frac{\partial \rho_{0} W^{e}}{\partial \underline{\underline{C^{e}}}}\left(\underline{\underline{C^{e}}}=\underline{\underline{C_{0}}}\right)$ where $\underline{\underline{C_{0}}}:={ }^{t} \underline{\underline{F_{0}}} \cdot \underline{\underline{F_{0}}}$ (See Figure 1). Note that here, the prestrain must be seen as a local tensor state variable more than a gradient of some mapping. And in this case, the full elastic transformation, $\underline{\underline{F^{e}}}$, contains both $\underline{\underline{F^{\prime}}}$ and the prestrain $\underline{\underline{F_{0}}}$ :

$$
\underline{\underline{F^{e}}}=\underline{\underline{F}} \cdot \underline{\underline{F_{0}}} \cdot{\underline{\underline{F^{g-1}}}}
$$


1 such that the free energy is

$$
\rho_{0} \Psi\left(\underline{\underline{C}}, \underline{\underline{F^{g}}}, \underline{\underline{F_{0}}}\right)=W^{e}\left(\underline{\underline{C^{e}}}={ }^{t} \underline{\underline{F^{g-1}}} \cdot{ }^{t} \underline{\underline{F_{0}}} \cdot \underline{\underline{C}} \cdot \underline{\underline{F_{0}}} \cdot \underline{\underline{F^{g-1}}}\right),
$$

2 and the second Piola-Kirchhoff stress tensor is

$$
\underline{\underline{\Sigma}}=\underline{\underline{F_{0}}} \cdot \underline{\underline{F^{g-1}}} \cdot 2 \frac{\partial W^{e}}{\partial \underline{\underline{C^{e}}}} \cdot{ }^{t} \underline{\underline{F^{g-1}}} \cdot{ }^{t} \underline{\underline{F_{0}}} .
$$

$3 \quad$ It is also interesting to express the prestrain in the grown configuration ${ }_{4} \Omega_{g}$ :

$$
\underline{\underline{F_{g 0}}}:=\underline{\underline{F^{g}}} \cdot \underline{\underline{F_{0}}} \cdot \underline{\underline{F^{g-1}}}
$$

5 such that the full elastic transformation can also be expressed as

$$
\underline{\underline{F^{e}}}=\underline{\underline{F^{\prime}}} \cdot \underline{\underline{F_{g 0}}}
$$

6 Similarly, the total prestrain in the new grown unloaded configuration $\Omega_{p}$ is

$$
\underline{\underline{F_{p 0}}}:=\underline{\underline{F^{p}}} \cdot \underline{\underline{F_{g 0}}}=\underline{\underline{F^{p}}} \cdot \underline{\underline{F^{g}}} \cdot \underline{\underline{F_{0}}} \cdot \underline{\underline{F^{g-1}}}
$$

7 such that the full elastic transformation can also be expressed as

$$
\underline{\underline{F^{e}}}=\underline{\underline{F^{l}}} \cdot \underline{\underline{F_{p 0}}}
$$

8 2.2. Relaxed growth modeling

9 2.2.1. Kinematics

10 We now describe a new formulation for finite growth, involving an ad11 ditional internal sub-transformation for local relaxation. The associated 12 schematic is represented on the Figure 2. The total transformation gradient 13 is now decomposed into growth, relaxation, prestrain and loading parts:

$$
\underline{\underline{F}}=\underline{\underline{F^{l}}} \cdot \underline{\underline{F^{p}}} \cdot \underline{\underline{F^{r}}} \cdot \underline{\underline{F^{g}}}
$$

where, again, both $\underline{\underline{F^{g}}}$ (the internal variable describing tissue growth), $\underline{\underline{F^{r}}}$ (the internal variable describing tissue relaxation) and $\underline{\underline{F^{p}}}$ (the prestrain) are incompatible second order tensor fields, and $\underline{\underline{F^{l}}}:=\underline{\underline{\operatorname{Grad}}}\left(\underline{\Phi^{l}}\right)$ is the gradient of the (compatible) transformation induced by mechanical loading. The elastic part of the transformation is still

$$
\underline{\underline{F^{\prime}}}:=\underline{\underline{F^{l}}} \cdot \underline{\underline{F^{p}}}
$$




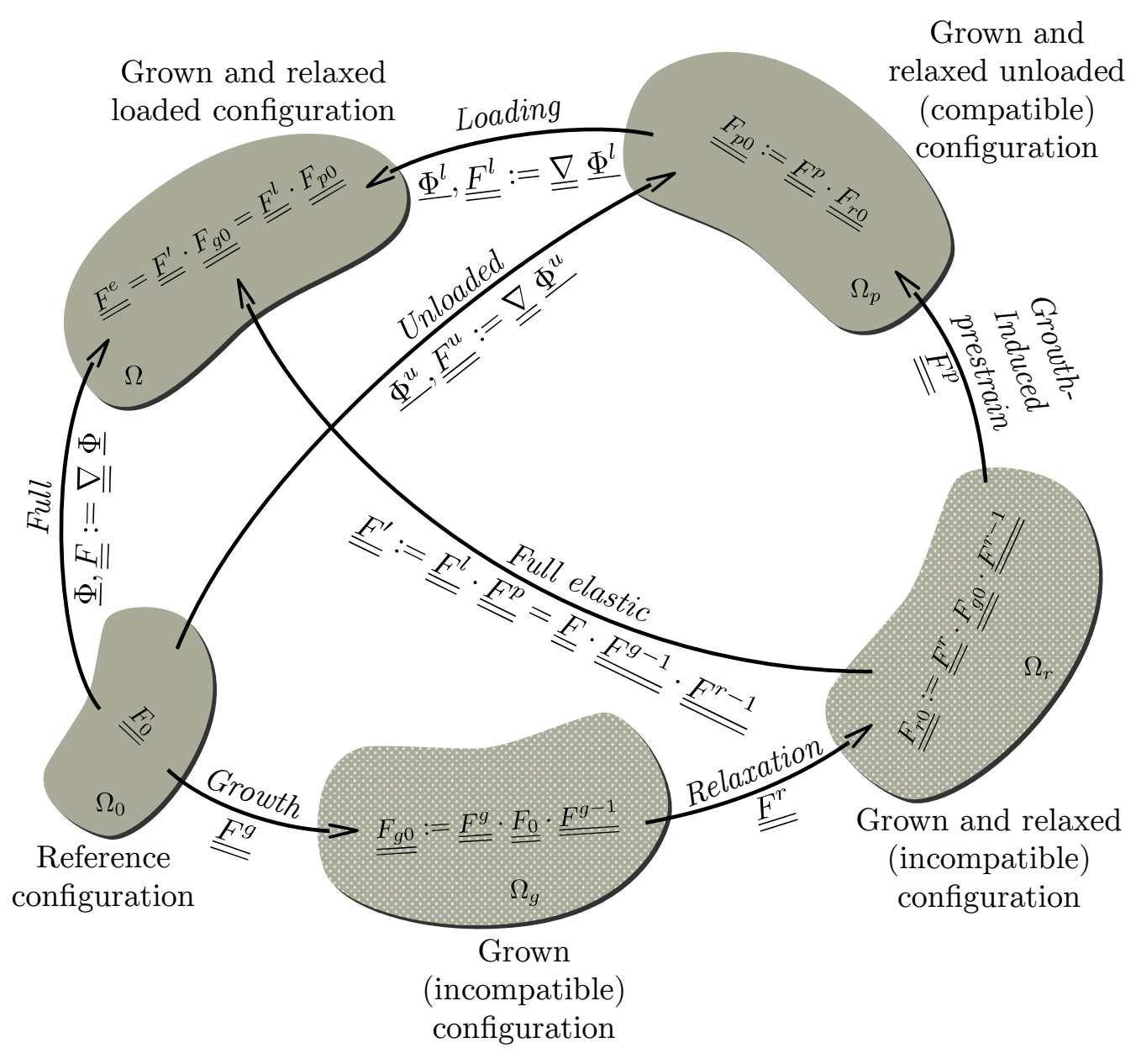

Figure 2: Schematic of the proposed multiplicative decomposition of the transformation gradient into growth, relaxation, prestrain and loading parts. 
such that

$$
\underline{\underline{F}}=\underline{\underline{F^{\prime}}} \cdot \underline{\underline{F^{r}}} \cdot \underline{\underline{F^{g}}} .
$$

2 Thus, here the elastic part of the transformation can be expressed from the 3 full transformation gradient, and both the growth and relaxation internal 4 variables:

$$
\underline{\underline{F^{\prime}}}:=\underline{\underline{F}} \cdot \underline{\underline{F^{g-1}}} \cdot \underline{\underline{F^{r-1}}} .
$$

The role of the relaxation is to update the unloaded configuration of the grown body in order to control the amount of growth-induced residual stresses. Thus, the updated unloaded configuration is now $\Omega_{p}$, with prestrain

$$
\underline{\underline{F_{p 0}}}:=\underline{\underline{F^{p}}} \cdot \underline{\underline{F_{r 0}}}
$$

where

$$
\underline{\underline{F_{r 0}}}:=\underline{\underline{F^{r}}} \cdot \underline{\underline{F_{g 0}}} \cdot \underline{\underline{F^{r-1}}}
$$

and

$$
\underline{\underline{F_{g 0}}}:=\underline{\underline{F^{g}}} \cdot \underline{\underline{F_{0}}} \cdot \underline{\underline{F^{g-1}}}
$$

\subsubsection{Free energy and Stresses}

As for simple growth, we assume that the free energy $\rho_{0} \Psi$ is only a function of the elastic part of the transformation $\underline{\underline{F^{e}}}$, see Equation (6). However, in the case of relaxed growth, we have

$$
\underline{\underline{F^{e}}}=\underline{\underline{F^{\prime}}} \cdot \underline{\underline{F_{r 0}}}=\underline{\underline{F}} \cdot \underline{\underline{F_{0}}} \cdot \underline{\underline{F^{g-1}}} \cdot \underline{\underline{F^{r-1}}}
$$

such that

$$
\underline{\underline{C^{e}}}={ }^{t} \underline{\underline{F}}^{-1} \cdot{ }^{t} \underline{\underline{F}}^{-1} \cdot{ }^{t} \underline{\underline{F_{0}}} \cdot \underline{\underline{C}} \cdot \underline{\underline{F_{0}}} \cdot \underline{\underline{F^{g-1}}} \cdot \underline{\underline{F^{r-1}}}
$$

and

$$
\underline{\underline{\Sigma}}=\underline{\underline{F_{0}}} \cdot \underline{\underline{F^{g-1}}} \cdot \underline{\underline{F^{r-1}}} \cdot 2 \frac{\partial W^{e}}{\partial \underline{\underline{C^{e}}}} \cdot{ }^{t} \underline{\underline{F^{r-1}}} \cdot \underline{\underline{F}}^{t-1} \cdot{ }^{t} \underline{\underline{F_{0}}} .
$$

Similarly, for the unloaded configuration the total elastic deformation is simply

$$
\underline{\underline{F_{p 0}}}=\underline{\underline{F^{u}}} \cdot \underline{\underline{F_{0}}} \cdot \underline{\underline{F^{g-1}}} \cdot \underline{\underline{F^{r-1}}}
$$

such that

$$
\underline{\underline{C_{p 0}}}:={ }^{t} \underline{\underline{F_{p 0}}} \cdot \underline{\underline{F_{p 0}}}={ }^{t} \underline{\underline{F^{r-1}}} \cdot{ }^{t} \underline{\underline{F^{g-1}}} \cdot{ }^{t} \underline{\underline{F_{0}}} \cdot \underline{\underline{C^{u}}} \cdot \underline{\underline{F_{0}}} \cdot{\underline{\underline{F^{g-1}}}} \cdot \underline{\underline{F^{r-1}}}
$$


and

$$
\underline{\underline{\Sigma^{u}}}=\underline{\underline{F_{0}}} \cdot \underline{\underline{F^{g-1}}} \cdot \underline{\underline{F^{r-1}}} \cdot 2 \frac{\partial \underline{W}^{e}}{\partial \underline{\underline{C_{p 0}}}} \cdot{ }^{t} \underline{\underline{F^{r-1}}} \cdot{ }^{t} \underline{\underline{F^{g-1}}} \cdot{ }^{t} \underline{\underline{F_{0}}} .
$$

\subsubsection{Relaxation evolution law}

3

$$
\underline{\underline{F^{r}}}=\frac{1}{\tau^{r}} \cdot \underline{\underline{E_{p 0}}}
$$

7 where $\tau^{r}$ is a characteristic time for relaxation, and $\underline{\underline{E_{p 0}}}:=\frac{1}{2}\left(\underline{\underline{C_{p 0}}}-\underline{\underline{1}}\right)$ is 8 the Green-Lagrange prestrain. The underlying hypothesis is that the tissue is 9 somehow able to sense the current level of prestrain. This is analogous to the

As for the growth evolution, many choices can be made for the evolution of the relaxation internal variable. Since the role of the relaxation is to regulate the prestrain, in this article we will consider the simplest possible evolution law, directly based on the current prestrain level: hypothesis made in constrained mixture theory-based growth models, where newly deposited matter has its own reference state that can be the one of the surrounding matter, the current state of deformation, or a combination of both [Valentín et al., 2013; Cyron et al., 2016].

\subsection{Numerical resolution}

The relaxed growth problem can be solved like any nonlinear problem with internal variables in mechanics. The only subtlety here is induced by the choice of the relaxation evolution law, which requires the computation of the new unloaded configuration together with the loaded configuration. We propose a mixed formulation of the problem, the unknowns being $\underline{U}$ (the total displacement), $\underline{U^{u}}$ (the displacement of the unloaded configuration), $\underline{\underline{F^{g}}}$ (the growth tensor) and $\underline{\underline{F^{r}}}$ (the relaxation tensor). After quasi-static assumption, and implicit, mid-point rule temporal discretization, the mixed 
1 variational formulation of the problem to solve at each time step is:

$$
\left\{\begin{array}{l}
\int_{\Omega_{0}} \underline{\underline{\Sigma}}: d_{\underline{U} ; \underline{U^{*}}} \underline{\underline{E}} d \Omega_{0}=\mathcal{W}_{e}\left(\underline{U} ; \underline{U^{*}}\right) \quad \forall \underline{\forall} \\
\int_{\Omega_{0}} \underline{\underline{\Sigma^{u}}}: d_{\underline{U^{u}} ; \underline{U^{u *}}} \underline{\underline{E^{u}}} d \Omega_{0}=0 \quad \forall \underline{U^{u *}} \\
\int_{\Omega_{0}}\left(\underline{\underline{F^{g}}}-\left(\underline{\underline{F^{g, \text { old }}}}+\underline{\underline{\dot{F}^{g, \text { mid }}}} \Delta t\right)\right): \underline{\underline{F^{g *}}} d \Omega_{0}=0 \quad \forall \underline{\underline{F^{g *}}} \\
\int_{\Omega_{0}}\left(\underline{\underline{F^{r}}}-\left(\underline{\underline{F^{r, \text { old }}}}+\underline{\underline{\dot{F}^{r, \text { mid }}}} \Delta t\right)\right): \underline{\underline{F^{r *}}} d \Omega_{0}=0 \quad \forall \underline{\underline{F^{r *}}}
\end{array}\right.
$$

2 where $d_{\underline{U ; U^{*}}} \underline{\underline{E}}$ and $d_{\underline{U^{u}} ; U^{u *}} \underline{\underline{E^{u}}}$ denote the first variations of the total and un3 loaded Green-Lagrange strain tensors with respect to the total and unloaded 4 displacements, and $\mathcal{W}_{e}$ denotes the virtual work (semi-linear form) associ-

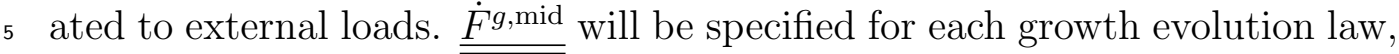
6 and relaxation evolution law $(31)$ leads to:

$$
\underline{\underline{\dot{F}^{r, \text { mid }}}}=\frac{1}{\tau^{r}} \underline{\underline{E_{p 0}^{\text {mid }}}}
$$

7 with $\underline{\underline{E_{p 0}^{\text {mid }}}}=\frac{1}{2}\left(\underline{\underline{E_{p 0}^{\text {old }}}}+\underline{\underline{E_{p 0}}}\right)$.

8 In case of incompressible elastic deformation, two new unknowns are 9 introduced: $p$ (the Lagrange multiplier associated to the incompressibility 10 constraint of the elastic strain, equal to the hydrostatic pressure within the tissue) and $p^{u}$ (the Lagrange multiplier/hydrostatic pressure in the unloaded configuration), and the full mixed variational formulation becomes:

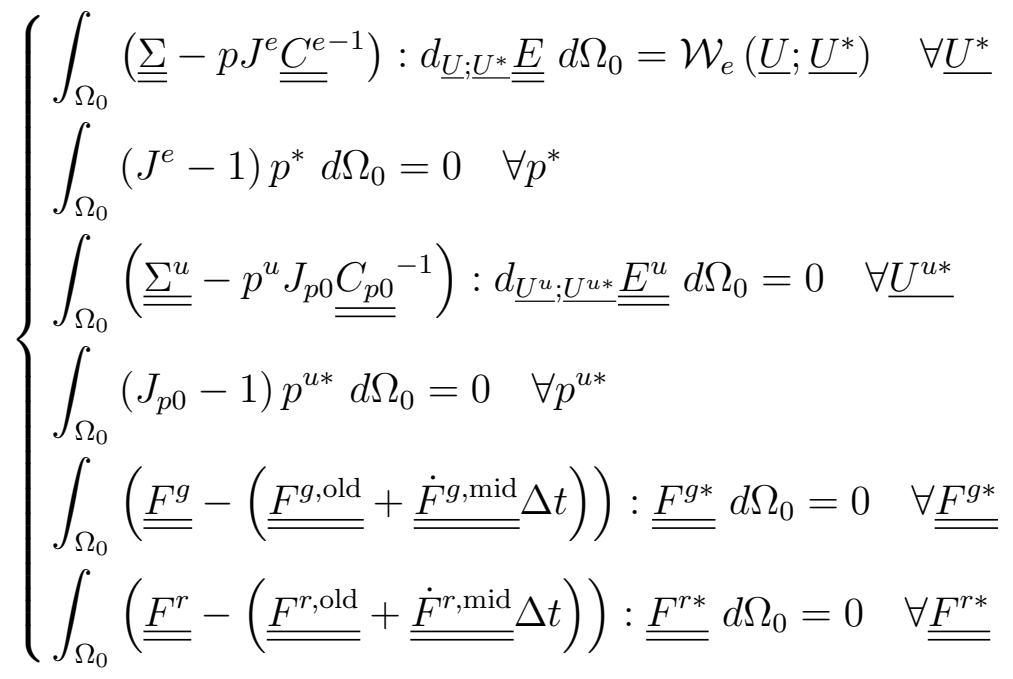


Problems (32) and (34) are spatially discretized using the standard finite element method. Second order elements are used for displacement unknowns (both $\underline{U} \& \underline{U^{u}}$ ), and first order elements for pressure unknowns (both $p$ $\& p^{u}$ ), preventing numerical locking in the incompressible limit [Hughes, 2000; Chapelle and Bathe, 2010]. First order elements are used for internal variables (both $\underline{\underline{F^{g}}} \& \underline{\underline{F^{r}}}$ ) as well.

The full scheme has been implemented in python, based on the FEniCS library [Logg et al., 2012; Alnæs et al., 2015], and is freely available ${ }^{1}$.

\section{Results}

We will now present multiple illustrations of the relaxed growth response in $2 \mathrm{D}$.

\subsection{Model response: constrained growth}

Let us first illustrate the constitutive behavior described by the relaxed growth model. To do so, we consider a single material point for which the total deformation is blocked to zero, and subjected to time-driven isotropic growth:

$$
\left\{\begin{array}{l}
\underline{\underline{F^{g}}}=\left(1+\theta^{g}\right) \underline{\underline{1}} \\
\dot{\theta}^{g}(t=0)=0
\end{array},\right.
$$

where $\tau^{g}$ is a characteristic time for growth. This growth evolution law leads, after temporal discretization, to the following expression:

$$
\underline{\underline{\dot{F}^{g, \text { mid }}}}=\frac{1}{\tau^{g}} \underline{\underline{1}}
$$

For the sake of simplicity, and to focus on the relaxed growth framework introduced in this paper, we consider a simple compressible neo-hookean strain energy potential under the plane strain assumption [Ciarlet and Geymonat, 1982]:

$$
W^{e}\left(\underline{\underline{C^{e}}}\right)=\frac{\lambda}{4}\left(J^{e 2}-1-2 \ln J^{e}\right)+\frac{\mu}{2}\left(\operatorname{tr}\left(\underline{\underline{C^{e}}}\right)-2-2 \ln J^{e}\right),
$$

\footnotetext{
${ }^{1}$ https://gitlab.inria.fr/mgenet/dolfin_cm
} 
where $\lambda \& \mu$ are the bulk and shear modulus, taken as unity. Compressible mixed variational formulation (32) is used, with evolution laws (33) \& (36).

This is the purest example of constrained growth. In case of simple growth, this would lead to the development of compressive residual stresses, which arise from constraining the grown tissue to its original volume. This is also what happens in the relaxed growth framework; however, here the amount of growth-induced stresses is controlled by the ratio of the characteristic time of relaxation to the characteristic time of growth. If relaxation is much slower than growth, the response is similar to simple growth; conversely, if relaxation is much faster than growth, then constrained growth happens with almost no induced residual stress. This is well illustrated on the Figure 3, which shows the normalized (with respect to shear modulus) hydrostatic pressure within the material point as a function of normalized (with respect to growth time constant) time, for various ratios of relaxation over growth characteristic times. The relaxed growth framework allows to control the amount of growth-induced residual stresses in constrained growth.

\subsection{A time-driven relaxed growth example: constrained vs. unconstrained growth}

Let us now illustrate the relaxed growth response in a first structural case, inspired from [Kuhl, 2014]. Here we consider a simple square geometry, with three fixed boundaries (left, bottom and right) and one free boundary (top), subjected to the same time-driven growth (35). This case contains both constrained (toward the bottom, where the total strain is restricted, thus developing compressive residual stresses), and unconstrained (toward the top, where the free edge limits the stress level, and large strains will develop) growth [Kuhl, 2014]. In terms of elasticity, we now consider a simple incompressible neo-hookean strain energy potential under the plane strain assumption:

$$
W^{e}\left(\underline{\underline{C^{e}}}\right)=\frac{\mu}{2}\left(\operatorname{tr}\left(\underline{\underline{C^{e}}}\right)-2\right),
$$

where $\mu$ is the shear modulus, taken as unity, and use the incompressible mixed variational formulation (34) is used with evolution laws (33) \& (36).

Figure 4 shows the response of the tissue modeled by relaxed growth, as a function of normalized (with respect to growth time constant) time, and for various ratios of relaxation over growth characteristic times. Since growth is purely time-driven, the growth pattern is very similar in all cases. However, 


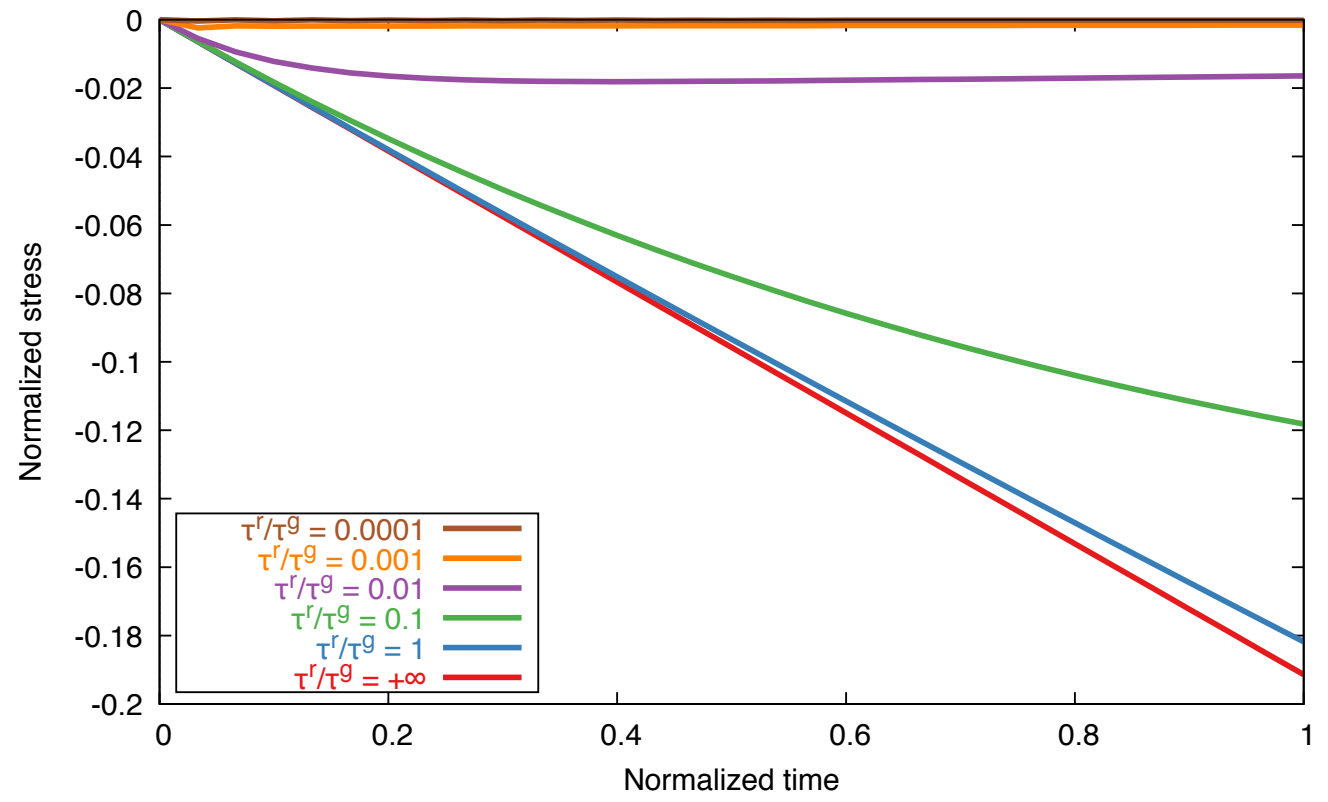

Figure 3: Relaxed growth material response under constrained growth: normalized (with respect to shear modulus) hydrostatic pressure within a material point as a function of normalized (with respect to growth time constant $\tau^{g}$ ) time, for various ratios of relaxation over growth characteristic times. For slow relaxation (i.e., for large relaxation time constant $\tau^{r}$ ), the model behaves as standard growth, with the development of residual stresses; conversely, for fast relaxation (i.e., for small relaxation time constant $\tau^{r}$ ), constrained growth happens with almost no induced residual stresses. 
the level of growth-induced residual stresses varies drastically with the relaxation characteristic time: for very fast relaxation (compared to growth), the muffin grows without developing large residual stresses, even in the constrained region.

\subsection{A strain-driven relaxed growth example: the artery}

The final example is the canonical case of residual stresses in pressurized arteries. We start from a simple disc representing an unloaded, stress-free artery. The artery is loaded with some internal pressure, and allowed to grow and relax for some time while the internal pressure is maintained. Then, the loading is removed, and an opening angle experiment is simulated by making a radial cut in the model, and letting the artery spring open by releasing some residual stresses. Here we consider the simplest form of strain-driven growth evolution law:

$$
\left\{\begin{array}{l}
\underline{\underline{F^{g}}}=\left(1+\theta^{g}\right) \underline{\underline{1}} \\
\theta^{g}(t=0)=0 \\
\dot{\theta}^{g}=\frac{\left\|\underline{\underline{E^{e}}}\right\|}{\tau^{g}}
\end{array}\right.
$$

where $\tau^{g}$ still represents the growth time constant, and $\underline{\underline{E^{e}}}:=\frac{1}{2}\left(\underline{\underline{C^{e}}}-\underline{\underline{1}}\right)$ is the Green-Lagrange elastic strain. This growth evolution law leads, after temporal discretization, to the following expression:

$$
\underline{\underline{\dot{F}^{g, \text { mid }}}}=\frac{\left\|\underline{\underline{E^{e, \text { mid }}}}\right\|}{\tau^{g}} \underline{\underline{1}}
$$

We use the incompressible neo-hookean strain energy potential (38), and the incompressible mixed variational formulation (34) with evolution laws (33) $\&(40)$.

Figure 5 shows the response of the artery over time, for various levels of relaxation characteristic times: it first inflates due to the applied pressure, grows, deflates as the pressure is removed, and springs open. Note that since residual stresses are here induced by heterogeneous, strain-driven growth described by growth evolution low (39), only a small amount of growth (with respect to the pressure-induced deformation) is required to generate realistic residual stresses, leading to physiological opening angles. This last example illustrates the fact that the relaxed growth model allows to control the opening angle (i.e., the residual stresses) induced by a given amount of growth. 


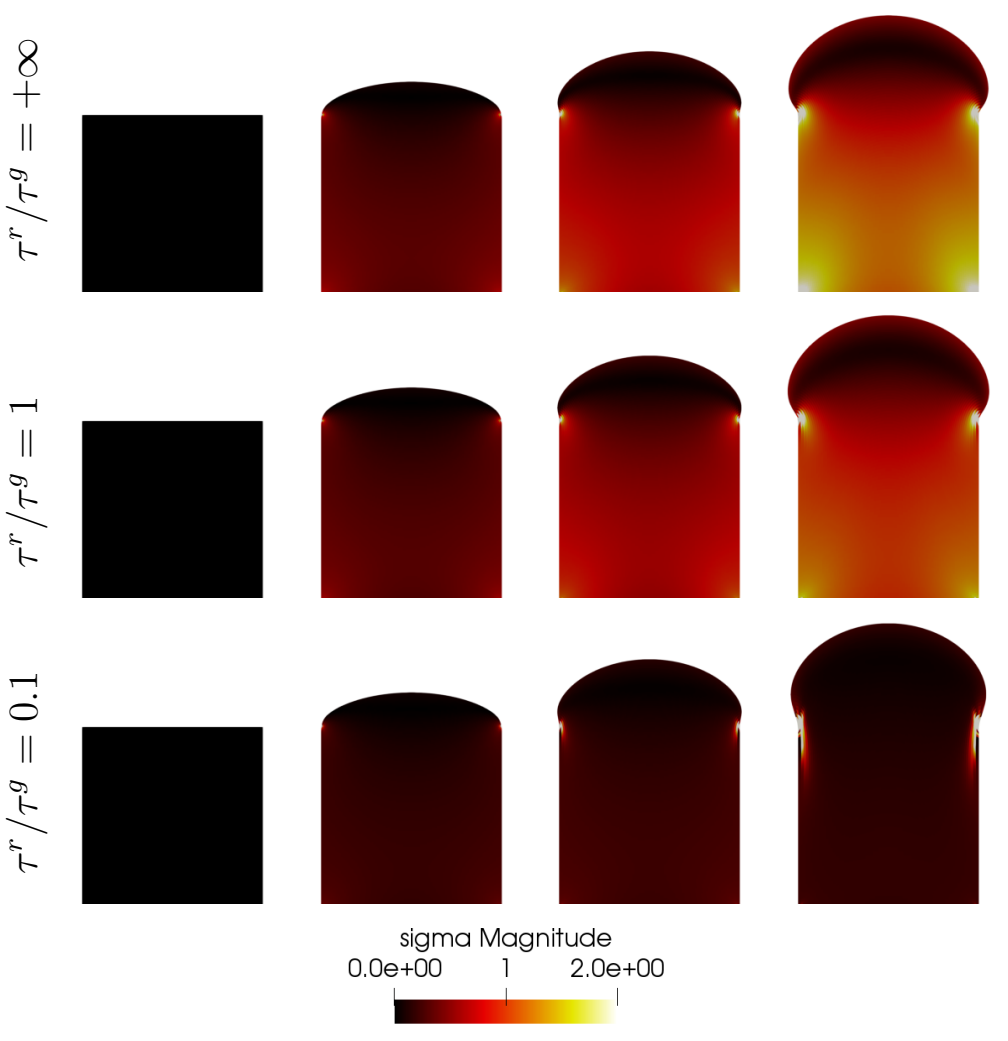

Figure 4: Relaxed growth structural response under constrained and unconstrained growth: norm of Cauchy stress tensor superimposed onto the deformed domain as a function of normalized (with respect to growth time constant $\tau^{r}$ ) time, for various ratios of relaxation over growth characteristic times. The faster the relaxation, the less residual stresses develop for the same amount of growth. 


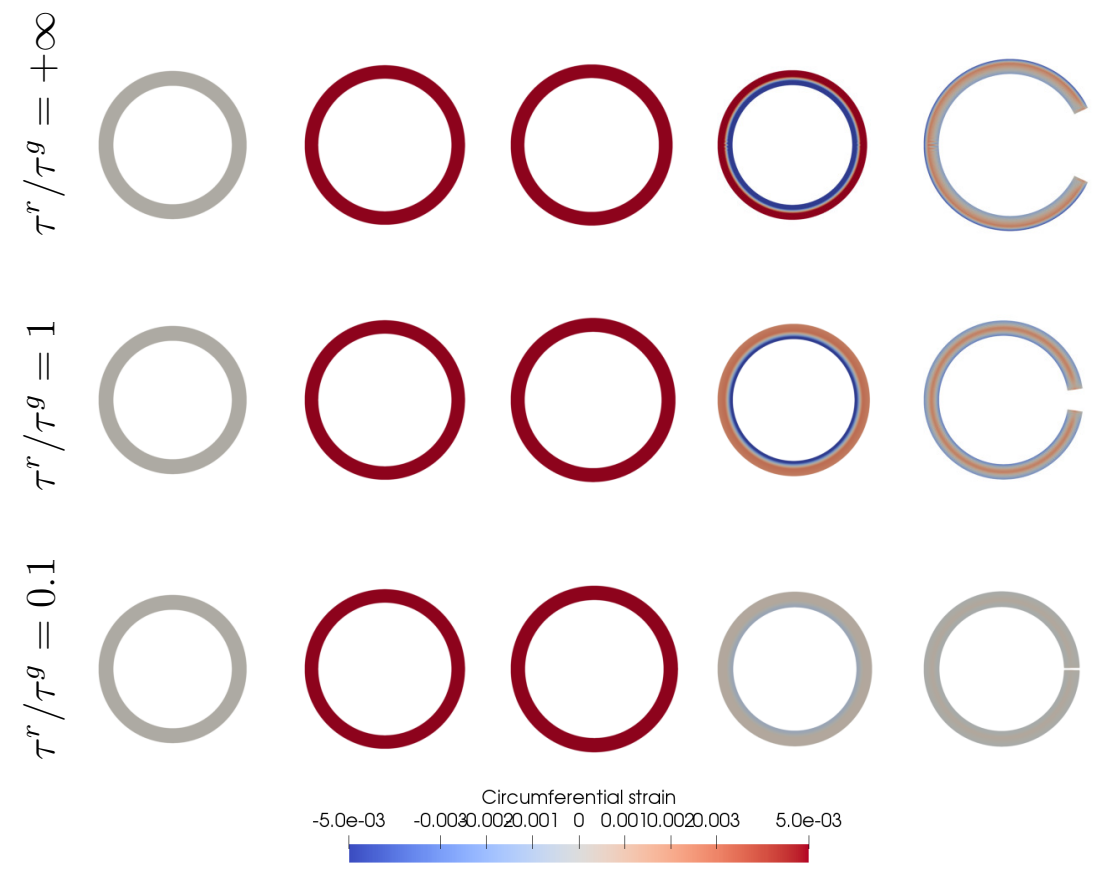

Figure 5: Relaxed growth response of a growing artery under pressure: circumferential strain superimposed onto the deformed artery at each step of the simulation (initial, after applying the internal pressure, after growth and relaxation, after removing the internal pressure, and after making a radial cut and letting the artery spring open), for various ratios of relaxation over growth characteristic times. The faster the relaxation, the less residual stresses, and hence a smaller opening angle develops for the same amount of growth. 


\section{Discussion}

In this paper, we introduced a novel "relaxed growth" framework allowing for a fine control of the amount of residual stresses generated during tissue heterogeneous growth. It is a direct extension of the classical multiplicative decomposition of the transformation gradient framework [Rodriguez et al., 1994], to which an additional sub-transformation is introduced in order to let the original unloaded configuration to evolve, hence relaxing away some of the residual stresses. To solve it numerically, we proposed here a monolithic mixed formulation, but a standard displacement formulation with internal variables could be used equivalently.

This work could be extended in multiple directions. Most and foremost, experimental characterization of the amount of residual stresses induced directly by physiological and/or pathological growth is required to provide elements of validation to relaxed growth models. Moreover, other relaxation evolution laws could be formulated and tested, especially in case of cyclic loading. 3D simulations could be run. More generally, since in the current numerical procedure both the deformed and unloaded configurations are computed at once, it is straightforward to stop the simulation, for instance when the total deformation becomes too important and the mesh too distorted, transfer all the fields to the new unloaded configuration, and restart from here. That way problems with extreme growth, for instance in organogenesis, could be tackled, with a clear control in both growth and growth-induced residual stresses. Finally, relaxed growth could be added personalized modeling pipelines, at both modeling and estimation steps, in order to make more objective and quantitative the handling of longitudinal clinical data, and design the next generation of diagnosis and treatment optimization tools.

\section{Acknowledgements}

MG would like to thank Prof. Lik Chuan Lee, from Michigan State University, USA, for helpful discussions throughout the development of this work.

\section{References}

Alnæs, M., Blechta, J., Hake, J., Johansson, A., Kehlet, B., Logg, A., Richardson, C., Ring, J., Rognes, M.E., Wells, G.N., 2015. The FEniCS Project Version 1.5. DOI:10.11588/ans. 2015.100.20553. 
Böl, M., Bolea Albero, A., 2014. On a new model for inhomogeneous volume growth of elastic bodies. Journal of the Mechanical Behavior of Biomedical Materials 29, 582-593. DOI:10.1016/j.jmbbm.2013.01.027.

Bolea Albero, A., Ehret, A.E., Böl, M., 2014. A new approach to the simulation of microbial biofilms by a theory of fluid-like pressure-restricted finite growth. Computer Methods in Applied Mechanics and Engineering 272, 271-289. DOI:10.1016/j.cma.2014.01.001.

Chapelle, D., Bathe, K.J., 2010. On the ellipticity condition for modelparameter dependent mixed formulations. Computers \& Structures 88, 581-587. DOI:10.1016/j.compstruc. 2010.01.009.

Ciarlet, P.G., Geymonat, G., 1982. Sur les lois de comportement en élasticité non-linéaire compressible. Comptes Rendus de l'Académie des Sciences Série II 295, 423-426.

Clatz, O., Sermesant, M., Bondiau, P.Y., Delingette, H., Warfield, S., Malandain, G., Ayache, N., 2005. Realistic simulation of the 3-D growth of brain tumors in MR images coupling diffusion with biomechanical deformation. IEEE Transactions on Medical Imaging 24, 1334-1346. DOI:10.1109/TMI.2005.857217.

Cyron, C.J., Aydin, R.C., Humphrey, J.D., 2016. A homogenized constrained mixture (and mechanical analog) model for growth and remodeling of soft tissue. Biomechanics and Modeling in Mechanobiology 15, 1389-1403. DOI:10.1007/s10237-016-0770-9.

Fung, Y.C., 1993. Biomechanics. Springer-Verlag, New York. DOI:10.1007/ 978-1-4757-2257-4.

Genet, M., Lee, L.C., Baillargeon, B., Guccione, J.M., Kuhl, E., 2016. Modeling Pathologies of Diastolic and Systolic Heart Failure. Annals of Biomedical Engineering 44, 112-127. DOI:10.1007/s10439-015-1351-2.

Genet, M., Lee, L.C., Ge, L., Acevedo-Bolton, G., Jeung, N., Martin, A.J., Cambronero, N., Boyle, A.J., Yeghiazarians, Y., Kozerke, S., Guccione, J.M., 2015a. A Novel Method for Quantifying Smooth Regional Variations in Myocardial Contractility Within an Infarcted Human Left Ventricle Based on Delay-Enhanced Magnetic Resonance Imaging. Journal of Biomechanical Engineering 137. DOI:10.1115/1.4030667. 
Genet, M., Rausch, M.K., Lee, L.C., Choy, S., Zhao, X., Kassab, G.S., Kozerke, S., Guccione, J.M., Kuhl, E., 2015b. Heterogeneous growthinduced prestrain in the heart. Journal of Biomechanics 48, 2080-2089. DOI:10.1016/j.jbiomech.2015.03.012.

5 Göktepe, S., Abilez, O.J., Kuhl, E., 2010. A generic approach towards finite growth with examples of athlete's heart, cardiac dilation, and cardiac wall thickening. Journal of the Mechanics and Physics of Solids 58, 1661-1680. DOI:10.1016/j.jmps.2010.07.003.

9 Holmes, J.W., 2004. Candidate mechanical stimuli for hypertrophy during volume overload. Journal of Applied Physiology 97, 1453-1460. DOI:10. 1152/japplphysiol.00834.2003.

Hughes, T.J.R., 2000. The Finite Element Method: Linear Static and Dynamic Finite Element Analysis. Dover Publications, Mineola, NY.

Humphrey, J.D., Rajagopal, K.R., 2002. A constrained mixture model for growth and remodeling of soft tissues. Mathematical Models and Methods in Applied Sciences 12, 407-430. DOI:10.1142/S0218202502001714.

Kerckhoffs, R.C.P., Omens, J.H., McCulloch, A.D., 2012. A single strainbased growth law predicts concentric and eccentric cardiac growth during pressure and volume overload. Mechanics Research Communications 42, 40-50. DOI:10.1016/j.mechrescom.2011.11.004.

Krishnamurthy, A., Villongco, C.T., Chuang, J., Frank, L.R., Nigam, V., Belezzuoli, E., Stark, P., Krummen, D.E., Narayan, S.M., Omens, J.H., McCulloch, A.D., Kerckhoffs, R.C.P., 2013. Patient-Specific Models of Cardiac Biomechanics. Journal of computational physics 244, 4-21. DOI:10.1016/j.jcp.2012.09.015.

Kroon, W., Delhaas, T., Arts, T., Bovendeerd, P.H.M., 2009. Computational modeling of volumetric soft tissue growth: Application to the cardiac left ventricle. Biomechanics and Modeling in Mechanobiology 8, 301-309. DOI:10.1007/s10237-008-0136-z.

Kuhl, E., 2014. Growing matter: A review of growth in living systems. Journal of the Mechanical Behavior of Biomedical Materials 29, 529-543. DOI:10.1016/j.jmbbm.2013.10.009. 
Lee, L.C., Genet, M., Acevedo-Bolton, G., Ordovas, K., Guccione, J.M., Kuhl, E., 2014. A computational model that predicts reverse growth in response to mechanical unloading. Biomechanics and Modeling in Mechanobiology 14, 217-229. DOI:10.1007/s10237-014-0598-0.

Lee, L.C., Sundnes, J.S., Genet, M., Wall, S.T., 2016. Physics-based computer simulation of the long-term effects of cardiac regenerative therapies. Technology 4, 23-29. DOI:10.1142/S2339547816400069.

Lee, L.C., Sundnes, J.S., Genet, M., Wenk, J.F., Wall, S.T., 2015. An integrated electromechanical-growth heart model for simulating cardiac therapies. Biomechanics and Modeling in Mechanobiology , 1-13DOI:10. 1007/s10237-015-0723-8.

Liu, S.Q., Fung, Y.C., 1989. Relationship between hypertension, hypertrophy, and opening angle of zero-stress state of arteries following aortic constriction. Journal of Biomechanical Engineering 111, 325-335. DOI:10.1115/1.3168386.

Logg, A., Mardal, K.A., Wells, G. (Eds.), 2012. Automated Solution of Differential Equations by the Finite Element Method: The FEniCS Book. Number 84 in Lecture Notes in Computational Science and Engineering, Springer, Heidelberg.

Maillet, M., van Berlo, J.H., Molkentin, J.D., 2013. Molecular basis of physiological heart growth: Fundamental concepts and new players. Nature reviews. Molecular cell biology 14, 38-48. DOI:10.1038/nrm3495.

Omens, J.H., Rodriguez, E.K., McCulloch, A.D., 1996. Transmural Changes in Stress-free Myocyte Morphology During Pressure Overload Hypertrophy in the Rat. Journal of Molecular and Cellular Cardiology 28, 1975-1983. DOI:10.1006/jmcc.1996.0190.

Rausch, M.K., Zöllner, A.M., Genet, M., Baillargeon, B., Bothe, W., Kuhl, E., 2017. A virtual sizing tool for mitral valve annuloplasty. International Journal for Numerical Methods in Biomedical Engineering 33, e02788. DOI:10.1002/cnm. 2788.

Rodriguez, E.K., Hoger, A., McCulloch, A.D., 1994. Stress-dependent finite growth in soft elastic tissues. Journal of Biomechanics 27, 455-467. 
Roth, C.J., Yoshihara, L., Ismail, M., Wall, W.A., 2017. Computational modelling of the respiratory system: Discussion of coupled modelling approaches and two recent extensions. Computer Methods in Applied Mechanics and Engineering 314, 473-493. DOI:10.1016/j.cma.2016.08. 010.

Sermesant, M., Chabiniok, R., Chinchapatnam, P., Mansi, T., Billet, F., Moireau, P., Peyrat, J.M., Wong, K.C.L., Relan, J., Rhode, K.S., Ginks, M.R., Lambiase, P.D., Delingette, H., Sorine, M., Rinaldi, C.A., Chapelle, D., Razavi, R., Ayache, N., 2012. Patient-specific electromechanical models of the heart for the prediction of pacing acute effects in CRT: A preliminary clinical validation. Medical Image Analysis 16, 201-15. DOI:10.1016/j . media.2011.07.003.

Skalak, R., Zargaryan, S., Jain, R.K., Netti, P.A., Hoger, A., 1996. Compatibility and the genesis of residual stress by volumetric growth. Journal of Mathematical Biology , 26.

Smith, N.P., de Vecchi, A., McCormick, M., Nordsletten, D.A., Camara, O., Frangi, A.F., Delingette, H., Sermesant, M., Relan, J., Ayache, N., Krueger, M.W., Schulze, W.H.W., Hose, R., Valverde, I., Beerbaum, P., Staicu, C., Siebes, M., Spaan, J., Hunter, P.J., Weese, J., Lehmann, H., Chapelle, D., Rezavi, R., 2011. euHeart: Personalized and integrated cardiac care using patient-specific cardiovascular modelling. Interface focus 1, 349-64. DOI:10.1098/rsfs. 2010.0048.

Taber, L.A., 1995. Biomechanics of Growth, Remodeling, and Morphogenesis. Applied Mechanics Reviews 48, 487. DOI:10.1115/1.3005109.

Taylor, C.A., Figueroa, C.A., 2009. Patient-specific modeling of cardiovascular mechanics. Annual Review of Biomedical Engineering 11, 109-134. DOI:10.1146/annurev . bioeng.10.061807.160521.

Valentín, A., Holzapfel, G.A., 2012. Constrained Mixture Models as Tools for Testing Competing Hypotheses in Arterial Biomechanics: A Brief Survey. Mechanics research communications 42, 126-133. DOI:10.1016/j . mechrescom.2012.02.003.

Valentín, A., Humphrey, J.D., Holzapfel, G.A., 2013. A finite element-based constrained mixture implementation for arterial growth, remodeling, and 
1 adaptation: Theory and numerical verification. International journal for 2 numerical methods in biomedical engineering 29, 822-49. DOI:10.1002/ 3 cnm. 2555.

4 Witzenburg, C.M., Holmes, J.W., 2017. A Comparison of Phenomenologic 5 Growth Laws for Myocardial Hypertrophy. Journal of Elasticity DOI:10. $6 \quad 1007 / \mathrm{s} 10659-017-9631-8$.

7 Xi, J., Lamata, P., Lee, J., Moireau, P., Chapelle, D., Smith, N.P., 2011. 8 Myocardial transversely isotropic material parameter estimation from in$9 \quad$ silico measurements based on a reduced-order unscented Kalman filter. 10 Journal of the mechanical behavior of biomedical materials 4, 1090-102. $11 \quad$ DOI:10.1016/j.jmbbm.2011.03.018. 